\title{
Runx1 is involved in primitive erythropoiesis in the mouse
}

Tomomasa Yokomizo ${ }^{1}$, Kazuteru Hasegawa $^{1}$, Hiroyuki Ishitobi ${ }^{1}$, Motomi Osato ${ }^{2}$, Masatsugu Ema ${ }^{1}$, Yoshiaki Ito ${ }^{2}$, Masayuki Yamamoto ${ }^{1,3}$ and Satoru Takahashi ${ }^{1,4}$

${ }^{1}$ Institute of Basic Medical Sciences, University of Tsukuba, 305-8575, Japan, ${ }^{2}$ Institute of Molecular and Cell Biology and Oncology Research Institute, 61Biopolis Drive, Proteos, Singapore 138673, Singapore, ${ }^{3}$ Center for Tsukuba Advanced Research Alliance, University of Tsukuba, 305-8575, Japan, ${ }^{4}$ Laboratory Animal Resource Center, University of Tsukuba, 305-8575, Japan.

T.Y. and K.H contributed equally to this study.

\section{Running Title}

Runx1 in primitive erythropoiesis.

Word count

Abstract: 126 words, Text words: 2231words

Corresponding author

Satoru Takahashi, M.D., PhD.

Institute of Basic Medical Sciences, University of Tsukuba

1-1-1 Tennoudai, Tsukuba 305-8575, Japan

Phone 81-29-853-7516; FAX 81-29-853-6965

E-mail: satoruta@md.tsukuba.ac.jp 


\section{Abstract}

Targeted disruption of the Runx1/ AML1 gene in mice has demonstrated that it is required for the emergence of definitive hematopoietic cells, but that it is not essential for the formation of primitive erythrocytes. These findings led to the conclusion that Runx1 is a stage-specific transcription factor acting only during definitive hematopoiesis. However, the zebrafish and Xenopus homologues of Runx1 have been shown to play roles in primitive hematopoiesis, suggesting that mouse Runx1 might also be involved in the development of primitive lineages. In this study, we show that primitive erythrocytes in $R u n x 1^{-/-}$mice display abnormal morphology and reduced expression of Ter119, Erythroid Kruppel-like factor (EKLF, KLF1), and GATA-1. These results suggest that mouse Runx1 plays a role in the development of both primitive and definitive hematopoietic cells. 


\section{Introduction}

Recent studies have revealed that the process of hematopoiesis is largely conserved in vertebrates. During development, similar sequential waves of primitive and definitive hematopoiesis are observed in mammals, fish, and amphibians. ${ }^{1-3}$ In the mouse embryo, primitive erythrocytes first appear in the yolk sac blood island at embryonic day (E) 7 and lead to the production of nucleated erythrocytes. ${ }^{4,5}$ These erythrocytes (erythroblasts) start to circulate at 4 to 6 somite pairs $(\mathrm{E} 8.25)^{6}$ and are necessary for the survival of the embryo before establishment of definitive erythropoiesis, which is initiated in the fetal liver around E12 and is characterized by the production of enucleated erythrocytes. ${ }^{4}$ The equivalent site of blood island in zebrafish embryo is known as the intermediate cell mass (ICM), which is located in the posterior part of embryo and provides the primitive erythrocytes. ${ }^{3}$

Runx1/AML1 encodes the DNA-binding subunit of the Runt domain transcription factor, ${ }^{7}$ which plays crucial roles in processes of organ and tumor development, such as hematopoiesis, neural development, and leukemogenesis. ${ }^{8,9}$ Runx1-deficient mice die around E12.5 due to severe hemorrhaging in the central nervous system (CNS) and a complete lack of definitive hematopoietic cells, including the aortic hematopoietic clusters, which are thought to comprise hematopoietic stem cells. ${ }^{10-14}$ The specification of definitive hematopoietic stem cells by Runx1 has also been observed in the zebrafish embryo. Depletion of zebrafish Runx1 by antisense 
morpholino oligonucleotides leads to a severe defect in definitive hematopoietic development in the dorsal aorta. ${ }^{15,16}$

Although Runx1 has been implicated in primitive hematopoiesis in zebrafish and Xenopus embryos, ${ }^{15,17}$ its function during primitive erythropoiesis in the mouse has not been described despite its reported expression in this lineage. ${ }^{13,18}$ In this study, we examined primitive erythrocytes in $R u n x 1^{-/-}$mice and observed aberrant morphology and reduced expression of Ter119, Erythroid Kruppel-like factor (EKLF, KLF1), and GATA-1, in these cells. 


\section{Materials \& Methods}

Mice

Runx1-deficient mice (C57BL/6) have been previously described. ${ }^{12}$ For visualizing GATA-1 expression in primitive erythrocytes, IE3.9-LacZ transgenic mice carrying a $3.9 \mathrm{~kb}$ GATA-1 promoter-LacZ reporter gene (C57BL/6 x DBA/2 mixed background $)^{19}$ were crossed with Runx1-deficient mice. For the transgenic complementary rescue experiment, G1-HRD-Runx1 transgenic mice carrying a $8.0 \mathrm{~kb}$ GATA-1 hematopoietic regulatory domain -Runx1 transgene (C57BL/6 x DBA/2 mixed background $)^{20}$ were crossed with Runx1-deficient mice.

\section{Benzidine- and LacZ-staining}

Embryos were fixed at $4^{\circ} \mathrm{C}$ for 2 hours in $1 \%$ formaldehyde, $0.2 \%$ glutaraldehyde, and $0.02 \%$ Nonidet P-40 in PBS. For LacZ-staining, embryos were incubated at $37^{\circ} \mathrm{C}$ overnight in $2 \mathrm{mM} \mathrm{MgCl}_{2}, 5 \mathrm{mM} \mathrm{K}_{3} \mathrm{Fe}(\mathrm{CN})_{6}, 5 \mathrm{mM} \mathrm{K}_{4} \mathrm{Fe}(\mathrm{CN})_{6}$, and $1 \mathrm{mg} / \mathrm{ml} \mathrm{X-Gal} \mathrm{in}$ PBS. For Benzidine-staining, embryos were incubated for $15 \mathrm{~min}$ in $14 \%$ Benzidine base ( $3 \%$ Benzidine in 90\% glacial acetic acid / $\left.10 \% \mathrm{H}_{2} 0\right)$ and $14 \% \mathrm{H}_{2} \mathrm{O}_{2}$ in $\mathrm{H}_{2} \mathrm{O}$.

\section{Scanning Electron Microscopy}

Peripheral blood cells from E12.5 embryos were fixed with 1.6\% glutaraldehyde, postfixed in $1 \%$ osmium tetraoxide. After dehydration with a graded series of alcohol 
solutions, the samples were freeze-dried, coated with a layer of gold and observed under a scanning electron microscope (JEOL, JSM-6320F).

\section{In vitro hematopoietic progenitor assay}

A colony assay for the development of primitive erythroid lineages was performed as described. ${ }^{21}$ Briefly, E8.5 whole embryos from each genotypes were treated with trypsin (Invitrogen) for $5 \mathrm{~min}$ at $37^{\circ} \mathrm{C}$, and washed with PBS containing $5 \%$ FBS. The cells were plated in 1\% methylcellulose/IMDM supplemented with $10 \%$ Plasma derived serum (PDS), 5\% Protein free hybridoma medium (PFHM) (Invitrogen), 12.5 $\mathrm{mg} / \mathrm{ml}$ ascorbic acid (Wako), $2 \mathrm{mM}$ L-glutamine, $10 \mathrm{mg} / \mathrm{ml}$ transferring (Boehringer Manheim), $39 \mathrm{ng} / \mathrm{ml}$ MTG (Sigma), $5 \mathrm{ng} / \mathrm{ml}$ stem cell factor (R\&D systems), 50 $\mathrm{ng} / \mathrm{ml}$ erythropoietin. After 3 days of culture, primitive erythroid colonies were counted.

\section{Cell preparation and staining}

Embryos were treated with Dispase II (Roche) and cell dissociation buffer containing EDTA/EGTA (Invitrogen) to make single cell suspensions. Non-specific binding was blocked by incubation in $5 \%$ mouse serum for $15 \mathrm{~min}$, followed by incubation with Oregon Green-conjugated anti-Ter119 antibody (a generous gift from Dr. S.-I. Nishikawa) for $30 \mathrm{~min}$. $\beta$-galactosidase activity was detected using FluoReporter lacZ 
Flow Cytometry Kit (Invitrogen). Briefly, dissociated cells were resuspended in $20 \mu 1$ of PBS with 5\% FCS prior to loading with $20 \mu \mathrm{l}$ of $2 \mathrm{mM}$ FDG (fluorescein di- $\beta$ -D-galactopyranoside) and followed by incubation at $37^{\circ} \mathrm{C}$ for $75 \mathrm{sec}$. The uptake was stopped by the addition of $500 \mu \mathrm{l}$ ice cold PBS with 5\% FCS. Cells were analyzed with FACScan or FACS Vantage (Becton Dickinson). Mean fluorescence intensity (MFI) values were calculated using CellQuest software (Becton Dickinson).

\section{Real-time RT-PCR}

RNA was purified from E11.5 peripheral blood cells using the RNeasy Kit (Qiagen) and reverse transcribed by SuperScriptIII (Invitrogen). Real-time polymerase chain reactions (PCR) employing Taqman probes for GATA-1 and GAPDH or with SYBR green for EKLF and SCL were performed in an ABI PRISM 7700 (Applied Biosystems). The forward and reverse primers used for PCR were as follows: EKLF, 5'-AGACTGTCTTACCCTCCATCAG-3' and 5'-GGTCCTCCGATTTCAGACTCAC-3'; SCL, 5'-CACTAGGCAGTGGGTTCTTTG-3' and 5'-GGTGTGAGGACCATCAGAAATCT-3'. The Primers and VIC-labeled probe for GATA-1 were as follows: 5'-CAGAACCGGCCTCTCATCC-3', 5'-TAGTGCATTGGGTGCCTGC-3', and VIC-labeled 5'-CCC AAG AAG CGA ATG ATT GTC AGC AAA-3'.$^{22}$ cDNA quantities were normalized to the amount of GAPDH cDNA, which was quantified using Rodent GAPDH Control Reagents (VIC 
probe, Applied Biosystems).

\section{Gene array analysis}

Total RNA from E10.5 wild type and $R u n x 1^{-/}$yolk sacs was extracted using RNeasy kit, and three same genotype samples were mixed equally. The quality and quantity of the isolated RNA was determined using an Agilent 2100 Bioanalyzer (Agilent Technologies). As previously described, ${ }^{23}$ Cy3- or Cy5-labeled cRNA was generated by the use of the Low RNA fluorescent Linear Amplification Kit (Agilent Technologies), and hybridized to a 20K Mouse Development Microarray (Agilent Technologies). The scanning was performed with an Agilent Technologies Microarray Scanner (Agilent Technologies), and the analysis was carried out using Excel software (Microsoft). 


\section{Results}

\section{Abnormal morphology of $\operatorname{Run} x \mathbf{1}^{-/-}$primitive erythrocytes}

To study the role of Runx1 in primitive erythropoiesis, we used a loss-of-function strategy. As reported previously, E10.5 Runx $1^{-/-}$embryos were not anemic and were indistinguishable from wild type embryos. ${ }^{10-12}$ Runx $1^{-/}$embryos began to hemorrhage in the CNS around E11.5, and died around E12.5. Firstly, to examine whether any morphological defects exist in Runx1-deficient primitive erythrocytes, we isolated peripheral blood from live E12.5 Runx $1^{-/-}$embryos. Cytospin preparation of $\operatorname{Run} x 1^{-/-}$peripheral blood revealed that $27 \%$ of primitive erythrocytes displayed a deformed shape of the erythrocytes, in contrast to the smooth, round shape of erythrocytes from $R u n x 1^{+-}$mice (Figure 1A-C). Consistently, scanning electron microscopic analysis revealed a rough surface and characteristic hole-like structures on $\operatorname{Runx1^{-/}}$ primitive erythrocytes (9 out of 20 erythrocytes, Figure 1G-I), not on Run $x 1^{+/-}$primitive erythrocytes ( 0 out of 16 erythrocytes, Figure $\left.1 \mathrm{D}-\mathrm{F}\right) . \quad$ As reported previously, ${ }^{18}$ the number of primitive erythroid colonies derived from $\operatorname{Run} x 1^{-/}$ embryos was similar to that derived from control Runx $1^{+/-}$embryos, indicating that formation of primitive erythroid progenitors is normal in Runx $1^{-/-}$embryos at E8.5 (Figure 2A). Benzidine staining of whole yolk sac revealed a normal number of

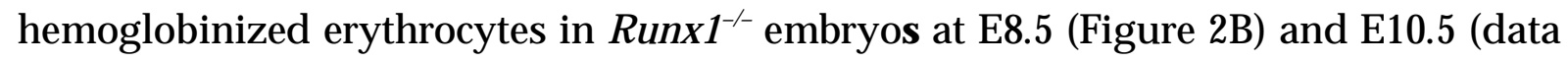
not shown). Taken together, these results suggest that Runx1 is required for the 
terminal maturation and/or maintenance of primitive erythrocytes.

\section{Reduced expression of Ter119 in $\operatorname{Runx} \mathbf{1}^{-/-}$primitive erythrocytes}

To further characterize $\operatorname{Run} x 1^{-/}$primitive erythrocytes, we analyzed the expression of the erythroid surface marker, Ter119, by fluorescence activated cell sorting (FACS). Compared with $R u n x 1^{+-}$embryos, Runx $1^{-/}$embryos show a reduction of Ter119 expression at E9.5 and E10.5 (Figure 2C and D), indicating that impairment of $R u n x 1^{-/-}$primitive erythrocytes already occurred at E9.5, at the molecular level.

\section{Microarray analysis of RNA prepared from the $\operatorname{Run} x \mathbf{1}^{-1-}$ yolk sac}

We next compared the gene expression profile of wild-type and $R u n x 1^{-/-}$embryos using microarray analysis to identify potential genes that function downstream of Runx1 in primitive erythropoiesis. For this experiment, we used the E10.5 yolk sac, since this organ at this stage is filled with primitive erythrocytes. We found that the expression of 43 genes was reduced in the $\operatorname{Run} x 1^{-/-}$yolk sac $(\mathrm{P}<0.01$, Table 1$)$. These included at least two genes encoding factors known to be involved in erythropoiesis, EKLF and $\alpha$-hemoglobin stabilizing protein $(A H S P) \cdot{ }^{24-26}$

\section{Reduced expression of EKLF and GATA-1 in Runx $\mathbf{1}^{-/}$embryos}

The expression of EKLF and AHSP is regulated by GATA-1, ${ }^{26,27}$ and knock-down of 
GATA-1 in embryonic stem cells leads to reduced expression of Ter $119,{ }^{28}$ leading us to speculate that GATA-1 expression might be affected by Runx1 depletion. GATA-1 is a critical regulator of both definitive and primitive erythrocyte differentiation. ${ }^{29}$ EKLF is also an important transcription factor involved in adult erythropoiesis and recently has been shown to be involved in the maintenance of both normal morphology and Ter119 expression in primitive erythrocytes. ${ }^{24,25}$ To quantify the expression of the erythroid transcription factors, GATA-1 and EKLF, in Runx $1^{-/-}$ embryos, we performed quantitative RT-PCR analysis. We also examined the expression of another erythroid transcription factor, SCL/Tal-1. Whereas the expression of SCL in E11.5 Runx1 $1^{--}$peripheral blood cells was comparable to that observed in Runx1 $1^{+-}$and wild type blood cells, the level of both GATA-1 and EKLF mRNAs was reduced by approximately $60 \%$ in $R u n x 1^{--}$mice (Figure 3A).

To confirm and better visualize the reduced expression of GATA-1 in Runx $1^{-/}$ primitive erythrocytes, we took advantage of mice transgenic for a GATA-1 promoter-LacZ hybrid gene construct (Figure 3B). We previously reported that a GATA-1 promoter element, $3.9 \mathrm{~kb}$ upstream from the GATA-1 proximal promoter (IE) exon, is active in primitive but not definitive erythrocytes. ${ }^{19}$ Thus, we introduced the GATA-1 promoter-LacZ (IE3.9-LacZ) transgene into Runx1 mutant mice as a surrogate to measure GATA-1 expression in $R u n x 1^{--}$primitive erythrocytes. Consistent with the RT-PCR analysis, expression of $\beta$-galactosidase from the 
transgene was reduced in E10.5 Runx $1^{-/-}$mice compared to $R u n x 1^{+/-}$mice (Figure 3C and D). Similar results were obtained in E8.5 (Figure 3C) and E11.5 embryos (data not shown). Reduced expression of $\beta$-galactosidase in $R u n x 1^{-/-}$mice was confirmed by FACS analysis (Figure 3E and F), suggesting that GATA-1 expression is dependent upon Runx1 in primitive erythrocytes.

\section{Transgenic rescue of $\operatorname{Run} x \mathbf{1}^{-/-}$primitive erythrocytes}

To determine whether erythroid-specific reintroduction of Runx1 rescues the abnormal phenotype of $R u n x 1^{-/}$primitive erythrocytes, transgenic mice expressing Runx1 under the control of the GATA-1 hematopoietic regulatory domain (G1-HRD, also called IE3.9int, Figure 4A) $)^{19,20}$ were crossed into the $R u n x 1^{-/-}$genetic background. The expression of GATA-1 was observed in the early erythroid progenitors, and G1-HRD significantly recapitulates the endogenous expression profile of GATA-1 in the erythroid lineage. ${ }^{19}$ Therefore, although GATA-1 expression was reduced by approximately $60 \%$ in $R u n x 1^{-/}$genetic background (Figure $3 \mathrm{~A}$ ), we assumed that this regulatory domain is suitable for our erythroid-specific rescue experiment. As expected, normal erythrocyte morphology and Ter119 expression were restored in Run $x 1^{-/-}$mice carrying the G1-HRD-Runx1 transgene (Figure 4B-D). Thus, we conclude that the defect in primitive erythrocytes in $R u n x 1^{-/}$mice is cell autonomous. 


\section{Discussion}

In this study, we demonstrated that $R u n x 1^{-/}$primitive erythrocytes exhibit some abnormal features. Impairment of primitive erythrocytes has also been observed in mice carrying a knocked-in $C B F \beta-M Y H 11$ gene. ${ }^{30} C B F \beta$ is a heterodimeric binding partner of Runx, and the fusion protein generated from the CBF $\beta-M Y H 11$ gene functions as a dominant negative inhibitor of Runx1 activity. ${ }^{31}$ In addition, although data were not shown, Wang and coworkers had noticed subtle abnormalities in the morphology and staining intensity of primitive erythrocytes from Run $x 1^{--}$embryos.

${ }^{11}$ These results further support our contention that Runx1 plays a role in primitive erythropoiesis in the mouse embryo.

Although the morphology of $\operatorname{Run} x 1^{--}$primitive erythrocytes is not normal, we believe that these erythrocytes are nonetheless able to deliver oxygen to body tissues based on following reasons. First, benzidine staining experiments demonstrated that erythrocytes in Runx $1^{-/}$embryos exhibit nearly normal levels of hemoglobinization (Figure 2B). Second, mutant mice lacking GATA-1 (Alas), which exhibit no functional primitive erythrocytes, die around E10.5, 32,33 indicating that intact primitive erythrocytes are essential for the survival of the embryo after E11. Unlike these latter mice, Runx1-deficient mice die instead around E12.5.

We showed here that expression of GATA-1 is at least partially dependent upon Runx1 expression in primitive erythrocytes (Figure 3). It is unknown, however, 
whether Runx1 directly regulates GATA-1 transcription. An interaction or association between Runx and GATA has been observed in other biological systems. Human RUNX1 and GATA-1 physically interact and synergistically activate the megakaryocyte gene in vitro. ${ }^{34}$ In the Drosophila blood system, coexpression of the GATA factor, Serpent, and the Runt-related factor, Lozenge, enhances crystal cell overproduction. ${ }^{35}$ Interestingly, mutations in GATA-1 were found in the vast majority of Down's syndrome (constitutional trisomy 21) patients with acute megakaryoblastic leukemia (DS-AMKL), and an extra copy of RUNX1 on chromosome 21 has been suspected to be responsible for the increased risk of DS-AMKL. ${ }^{36}$ These observations suggest that Runx and GATA factors work cooperatively and may modulate one another's activity in a gene regulatory network.

We have uncovered a previously unrecognized role for Runx1 in mouse primitive erythropoiesis. Runx1 is also expressed during immature stages of definitive erythrocytes. ${ }^{37}$ In addition, impairment of definitive erythroid differentiation has been observed in an AML patient with a $t(8 ; 21)$ translocation, which results in the production of an AML1-ETO fusion protein. ${ }^{38}$ These observations suggest that Runx1 may play a role in definitive erythropoiesis. Although there is a report that there is no apparent reduction of the hemoglobin level in Runx1-deficient peripheral blood cells, ${ }^{39}$ it is worth examining more extensively whether conditional loss of Runx1 in erythroid lineage influences adult 
erythropoiesis. 


\section{Acknowledgements}

We would like to thank Dr. S.-I. Nishikawa for reagents and Dr. O. Nakajima for protocol. 


\section{References}

1. Dzierzak E. Hematopoietic stem cells and their precursors: developmental diversity and lineage relationships. Immunol Rev. 2002;187:126-138

2. Ciau-Uitz A, Walmsley M, Patient R. Distinct origins of adult and embryonic blood in Xenopus. Cell. 2000;102:787-796

3. Davidson AJ, Zon L. The 'definitive' (and 'primitive') guide to zebrafish hematopoiesis. Oncogene. 2004;23:7233-7246

4. Palis J, Yoder MC. Yolk-sac hematopoiesis: the first blood cells of mouse and man. Exp Hematol. 2001;29:927-936

5. Ema M, Yokomizo T, Wakamatsu A, Terunuma T, Yamamoto M, Takahashi S. Primitive erythropoiesis from mesodermal precursors expressing VE-cadherin, PECAM-1, Tie2, endoglin, and CD34 in the mouse embryo. Blood. 2006;108:4018-4024 6. McGrath KE, Koniski AD, Malik J, Palis J. Circulation is established in a stepwise pattern in the mammalian embryo. Blood. 2003;101:1669-1676

7. Ito Y. Molecular basis of tissue-specific gene expression mediated by the runt domain transcription factor PEBP2/CBF. Genes Cells. 1999;4:685-696

8. Speck NA, Gilliland DG. Core-binding factors in haematopoiesis and leukaemia. Nature Reviews Cancer. 2002;2:502-513

9. Yoshikawa M, Senzaki K, Yokomizo T, Takahashi S, Ozaki S, Shiga T. Runx1 selectively regulates cell fate specification and axonal projections of dorsal root 
ganglion neurons. Dev. Biol. 2007;303:663-674

10. Okuda T, van Deursen J, Hiebert SW, Grosveld G, Downing JR. AML1, the target of multiple chromosomal translocations in human leukemia, is essential for normal fetal liver hematopoiesis. Cell. 1996;84:321-330

11. Wang $\mathrm{Q}$, Stacy $\mathrm{T}$, Binder $\mathrm{M}$, et al. Disruption of the Cbfa2 gene causes necrosis and hemorrhaging in the central nervous system and blocks definitive hematopoiesis. Proc. Natl. Acad. Sci. USA. 1996;93:3444-3449

12. Okada H, Watanabe T, Niki M, et al. AML1(-/-) embryos do not express certain hematopoiesis-related gene transcripts including those of the PU.1 gene. Oncogene. $1998 ; 17: 2287-2293$

13. North $\mathrm{T}, \mathrm{Gu} \mathrm{TL}$, Stacy $\mathrm{T}$, et al. Cbfa2 is required for the formation of intra-aortic hematopoietic clusters. Development. 1999;126:2563-2575

14. Yokomizo $\mathrm{T}$, Ogawa $\mathrm{M}$, Osato $\mathrm{M}$, et al. Requirement of Runx1/AML1/PEBP2 $\alpha$ B for the generation of haematopoietic cells from endothelial cells. Genes Cells. 2001;6:13-23

15. Kalev-Zylinska ML, Horsfield JA, Flores MV, et al. Runx1 is required for zebrafish blood and vessel development and expression of a human RUNX1-CBF2T1 transgene advances a model for studies of leukemogenesis. Development. 2002;129:2015-2030

16. Burns CE, Traver D, Mayhall E, Shepard JL, Zon LI. Hematopoietic stem cell 
fate is established by the Notch-Runx pathway. Genes Dev. 2005;19:2331-2342

17. Tracey WD Jr, Pepling ME, Horb ME, Thomsen GH, Gergen JP. A Xenopus homologue of aml-1 reveals unexpected patterning mechanisms leading to the formation of embryonic blood. Development. 1998;125:1371-1380

18. Lacaud G, Gore L, Kennedy M, et al. Runx1 is essential for hematopoietic commitment at the hemangioblast stage of development in vitro. Blood. 2002;100:458-466

19. Onodera K, Takahashi S, Nishimura S, et al. GATA-1 transcription is controlled by distinct regulatory mechanisms during primitive and definitive erythropoiesis. Proc Natl Acad Sci USA. 1997;94:4487-4492

20. Yokomizo T, Takahashi S, Mochizuki N, et al. Characterization of GATA-1 ${ }^{+}$ hemangioblastic cells in the mouse embryo. EMBO J. 2007;26:184-196

21. Palis J, Robertson S, Kennedy M, Wall C, Keller G. Development of erythroid and myeloid progenitors in the yolk sac and embryo proper of the mouse.

Development. 1999;126:5073-5084

22. Suzuki N, Suwabe N, Ohneda O, et al. Identification and characterization of 2 types of erythroid progenitors that express GATA-1 at distinct levels. Blood. $2003 ; 102: 3575-83$

23. Suzuki Y, Nakayama M. Differential profiles of gene expressed in neonatal brain of $129 \times 1 / \mathrm{SvJ}$ and C57BL/6J mice: a database to aid in analyzing DNA 
microarrays using nonisogenic gene-targeted mice. DNA Research. 2003;10:263-275

24. Drissen R, von Lindern M, Kolbus A, et al. The erythroid phenotype of EKLF-null mice: defects in hemoglobin metabolism and membrane stability. Mol. Cell. Biol. 2005;25:5205-5214

25. Hodge D, Coghill E, Keys J, et al. A global role for EKLF in definitive and primitive erythropoiesis. Blood. 2006;107:3359-3370

26. Kihm AJ, Kong Y, Hong W, et al. An abundant erythroid protein that stabilizes free alpha-haemoglobin. Nature 2002;417:758-763

27. Anderson KP, Crable SC, Lingrel JB. Multiple proteins binding to a GATA-E box-GATA motif regulate the erythroid Kruppel-like factor (EKLF) gene. J. Biol. Chem. 1998;273:14347-14354

28. Suwabe N, Takahashi S, Nakano T, Yamamoto M. GATA-1 regulates growth and differentiation of definitive erythroid lineage cells during in vitro ES cell differentiation. Blood. 1998;92:4108-4118

29. Ferreira R, Ohneda K, Yamamoto M, Philipsen S. GATA1 Function, a Paradigm for Transcription Factors in Hematopoiesis. Mol Cell Biol. 2005;25:1215-1227

30. Castilla LH, Wijmenga C, Wang Q, et al. Failure of embryonic hematopoiesis and lethal hemorrhages in mouse embryos heterozygous for a knocked-in leukemia gene CBFB-MYH11. Cell. 1996;87:687-696

31. Huang G, Shigesada K, Wee HJ, Liu PP, Osato M, Ito Y. Molecular basis for a 
dominant inactivation of RUNX1/AML1 by the leukemogenic inversion 16 chimera. Blood. 2004;103:3200-3207

32. Fujiwara Y, Browne CP, Cunniff K, Goff SC, Orkin SH. Arrested development of embryonic red cell precursors in mouse embryos lacking transcription factor GATA-1. Proc. Natl. Acad. Sci. U S A. 1996;93:12355-12358

33. Nakajima $\mathrm{O}$, Takahashi $\mathrm{S}$, Harigae $\mathrm{H}$, et al. Heme deficiency in erythroid lineage causes differentiation arrest and cytoplasmic iron overload. EMBO J. $1999 ; 18: 6282-6289$

34. Elagib KE, Racke FK, Mogass M, Khetawat R, Delehanty LL, Goldfarb AN. RUNX1 and GATA-1 coexpression and cooperation in megakaryocytic differentiation. Blood. 2003;101:4333-4341

35. Fossett N, Hyman K, Gajewski K, Orkin SH, Schulz RA. Combinatorial interactions of serpent, lozenge, and U-shaped regulate crystal cell lineage commitment during Drosophila hematopoiesis. Proc. Natl. Acad. Sci. U S A. 2003;100:11451-11456.

36. Gurbuxani S, Vyas P, Crispino JD. Recent insights into the mechanisms of myeloid leukemogenesis in Down syndrome. Blood. 2004;103:399-406

37. North TE, Stacy T, Matheny CJ, Speck NA, de Bruijn MF. Runx1 is expressed in adult mouse hematopoietic stem cells and differentiating myeloid and lymphoid cells, but not in maturing erythroid cells. Stem Cells. 2004;22:158-168 
38. Yamasaki H, Era T, Asou N, et al. High degree of myeloid differentiation and granulocytosis is associated with $t(8 ; 21)$ smoldering leukemia. Leukemia. 1995;9:1147-1153.

39. Ichikawa M, Asai T, Saito $\mathrm{T}$, et al. AML-1 is required for megakaryocytic maturation and lymphocytic differentiation, but not for maintenance of hematopoietic stem cells in adult hematopoiesis. Nat Med. 2004;10:299-304 


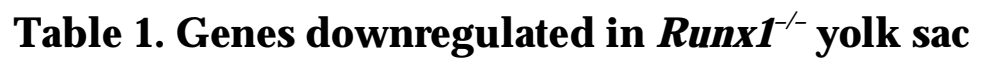

\begin{tabular}{|c|c|c|c|c|c|}
\hline $\begin{array}{l}\text { Systematic } \\
\text { Name }\end{array}$ & Gene & Symbol & $\begin{array}{c}\text { Wild-type } \\
\text { signal }\end{array}$ & $\begin{array}{c}\text { Runx1 KO } \\
\text { signal }\end{array}$ & $\begin{array}{l}\text { Fold } \\
\text { Change }\end{array}$ \\
\hline H3044F08-3 & Mitogen inducible mig & MGC36836 & 3362.7 & 767.1 & 0.2281 \\
\hline L0240C12-3 & Complement component 1, q subcomponent, alpha polypeptide & C1qa & 3672.5 & 1220.3 & 0.3323 \\
\hline L0075B01-3 & Mus domesticus strain MilP mitocondrion genome, complete sequence & - & 30184.8 & 14344.7 & 0.4752 \\
\hline H3054H09-3 & $\mathrm{H} 3054 \mathrm{H} 09-3$ & - & 7727.4 & 4002.2 & 0.5179 \\
\hline M97200.1 & Erythroid Kruppel-like factor & EKLF & 4482.6 & 2440.6 & 0.5445 \\
\hline C0284A02-3 & Hydroxysteroid dehydrogenase-1, delta $<5>-3$-beta & Hsd3b1 & 3796.8 & 2093.6 & 0.5514 \\
\hline L0258G12-3 & Muscleblind-like & Mbnl & 5640.8 & 3188.8 & 0.5653 \\
\hline H3126G01-5 & H3126G01-5 & - & 82880.5 & 47827.7 & 0.5771 \\
\hline H3060Е04-3 & Protein kinase $\mathrm{C}$ type EC2.7.1- & 6030436C20Rik & 3320.5 & 1954.3 & 0.5886 \\
\hline H3114C08-3 & 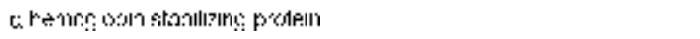 & AHSP & 8460.8 & 5144.7 & 0.6081 \\
\hline H3087E02-3 & riken cDNA 4933436C10 gene & 4933436C10Rik & 9907.1 & 6159.5 & 0.6217 \\
\hline H3059D10-3 & Cornichon homolog & Cnih & 11741.2 & 7310.0 & 0.6226 \\
\hline J0460E10-3 & J0460E10-3 & - & 5814.1 & 3646.0 & 0.6271 \\
\hline H3048G11-3 & Biliverdin reductase $B$ & Blvrb & 10690.0 & 6713.2 & 0.6280 \\
\hline H3065D10-3 & RAS-related protein-1a & Rap1a & 19757.3 & 12435.3 & 0.6294 \\
\hline L0222E01-3 & Rhesus blood group CE and D & Rhced & 10397.4 & 6590.9 & 0.6339 \\
\hline H3056A02-3 & H3056A02-3 & - & 4661.2 & 2981.7 & 0.6397 \\
\hline H3157F03-3 & riken cDNA 2810465016 gene & 2810465016Rik & 5012.1 & 3214.5 & 0.6414 \\
\hline $\mathrm{C} 0321 \mathrm{H} 12-3$ & Cell growth regulator & 1110038G02Rik & 8733.7 & 5608.6 & 0.6422 \\
\hline H3076H08-3 & $\mathrm{H} 3076 \mathrm{H} 08-3$ & - & 20849.6 & 13503.4 & 0.6477 \\
\hline C0166A10-3 & Carbonic anhydrase 2 & Car2 & 27887.6 & 18109.0 & 0.6494 \\
\hline
\end{tabular}

Genes downregulated less than 0.65-fold in Runx1-deficient yolk sacs were listed. Probe informations and gene annotations of Agilent Mouse Development Microarrays are available at the NIA mouse cDNA project home page (http://lgsun.grc.nia.nih.gov/cDNA/cDNA.html). 


\section{Figure legends}

Figure 1. $\operatorname{Runx1^{-/}}$ primitive erythrocytes show defect in morphology. (A, B) May-Grunwald Giemsa stained cytospin preparation of peripheral blood cells at E12.5. While normal erythrocytes (arrowhead) can be seen in $R u n x 1^{-/}$embryo, $27 \%$ of them show apparent deformed shape (arrows). (C) Quantification of abnormal primitive erythrocytes in $R u n x 1^{+--}(n=3)$ and $R u n x 1^{-/-}(n=4)$ embryos at E12.5. Asterisk indicates significance, $\mathrm{P}=0.002$, Student $t$ test. (D-I) Scanning electron microscopy of primitive erythrocytes at E12.5. Shown are representative pictures of primitive erythrocytes from $R u n x 1^{+/}(\mathrm{n}=3)$ and $R u n x 1^{-/-}(\mathrm{n}=3)$ embryos. Characteristic large holes (white arrowheads) were observed in Runx $1^{-/-}$erythrocytes (9/20), not in Run $x 1^{+/-}$erythrocytes $(0 / 16)$. This difference is statistically significant $(\mathrm{P}=0.002$ by Fisher's exact probability test). Original magnifications are x 400 for A and B, x 8000 for D, E, F and G, x 8500 for $\mathrm{H}$, and $\times 9000$ for $\mathrm{I}$.

Figure 2. Analysis of primitive erythroid proginitors and differentiated cells in $\boldsymbol{R u n x}^{-/-}$embryo. (A) Primitive erythroid colonies from E8.5 Runx $1^{+/-}$yolk sacs $(\mathrm{n}=3)$ and Run $x 1^{-/}$yolk sacs $(\mathrm{n}=3)$. (B, C) Benzidine-staining of E8.5 embryos. Hemoglobinized cells were observed inside blood vessels of yolk sac. Similar staining levels were obtained for wild type and $R u n x 1^{-/}$embryos. Original magnifications are $x$ 16.0. (D) Flow cytometric analysis of E9.5 yolk sac using 
anti-Ter119 antibody. Black line, gray bold line, or red bold line represent Ter119 expression in $R u n x 1^{+/+}, R u n x 1^{+/}$, and $R u n x 1^{-/-}$, respectively. (E) Relative MFI values of

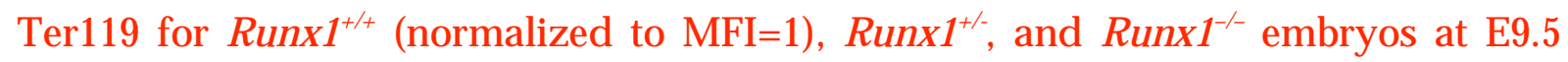
and E10.5. Bar graphs represents mean ratios \pm standard deviations derived from $\operatorname{Runx}^{+-}(\mathrm{n}=3 \mathrm{E} 9.5, \mathrm{n}=4 \mathrm{E} 10.5)$ and $\operatorname{Runx}^{-/-}(\mathrm{n}=2 \mathrm{E9} .5, \mathrm{n}=4$ E10.5) embryos. Significant differences in $R u n x 1^{-/}$mice from $R u n x 1^{+-}$mice were observed; ${ }^{* *} P=0.017,{ }^{*} P=0.039$ by Student $t$ test.

Figure 3. Reduced expression of GATA-1 and EKLF in $R u n x 1^{-1-}$ primitive erythrocytes. (A) Relative intensity levels were measured by real-time PCR and normalized to GAPDH. The Student $t$ test was applied for statistical analysis. Significant differences in $R u n x 1^{-/}$mice from wild type were observed; ${ }^{* *} P=0.009$, ${ }^{*} P=0.013$. (B) Structure of the GATA-1 promoter-LacZ (IE3.9-LacZ) transgene. IE indicates exon IE. (C) Representative pictures of Runx1 $1^{+-:: I E 3.9-L a c Z ~(n=7 ~ E 8.5, ~ n=12 ~}$ E10.5) and Runx1 $1^{--}:$IE3.9-LacZ ( $\mathrm{n}=8$ E8.5, $\mathrm{n}=4$ E10.5) embryos. Expression of $\beta$-galactosidase was reduced in $R u n x 1^{-/}$embryo (C, right panels) compared to Run $x 1^{+-}$embryo (C, left panels). (D) Cytospin preparation of blood cells from embryos shown in panel C (E10.5). Intensity of $\beta$-galactosidase staining was reduced in $R u n x 1^{-/}$cells (arrowheads) compared to Run $x 1^{+-}$cells (arrows). (E) FACS analysis of GATA-1 ( $\beta$-galactosidase) expression in E10.5 embryos. FDG was used as a 
fluorescent substrate for $\beta$-galactosidase. Black line, gray bold line, or red bold line represent $\beta$-galactosidase expression in $R u n x 1^{+/+}, R u n x 1^{+/}$, and $R u n x 1^{-/}$, respectively. (F) MFI values of FDG for $R u n x 1^{+/+}(\mathrm{n}=2) \operatorname{Run} x 1^{+-}(\mathrm{n}=5)$ and $R u n x 1^{-/-}(\mathrm{n}=2)$ embryos. Significant difference in $R u n x 1^{-/-}$mice from $R u n x 1^{+-}$mice was observed; ${ }^{*} P=0.032$ by Student $t$ test. Original magnifications are $\mathrm{x} 16.0$ for $\mathrm{C}$ (upper panels), $\mathrm{x} 7.2$ for $\mathrm{C}$ (lower panels), and x 400 for D.

Figure 4. Rescue of defects in $\operatorname{Run} x 1^{-/-}$primitive erythrocytes by transgenic introduction of Runx1. (A) Structure of the G1-HRD-Runx1 (IE3.9int-Runx1) transgene. IE and II indicate exon IE and II, respectively. (B) Rescue of Ter119 expression on E10.5 Runx $1^{-/-}$primitive erythrocytes by introducing G1-HRD-Runx1 transgene. Black line, gray bold line, or red bold line represent Ter119 expression in Runx $1^{+/+}$, Runx $1^{--}::$G1-HRD-Runx1 and Runx $1^{-/}$, respectively. Note that overexpression of Runx1 in $R u n x 1^{-/-}$erythrocytes induced slightly higher Ter119 expression. (C) Relative MFI values of Ter119 for $R u n x 1^{+/+}$(normalized to MFI=1), Runx $x 1^{-1}$, and Runx $1^{-1}:: G 1-H R D-R u n x 1$ embryos at E10.5. Bar graphs represents mean

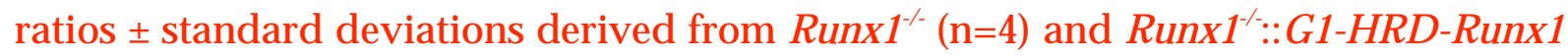
$(\mathrm{n}=4)$ embryos. Significant difference in $R u n x 1^{--}:: G 1-H R D-R u n x 1$ mice from $R u n x 1^{--}$ mice was observed; ${ }^{*} P<0.001$ by Student $t$ test. (D) Rescue of abnormal morphology observed in $R u n x 1^{-/-}$primitive erythrocytes. May-Grunwald Giemsa stained cytospin 
preparation of peripheral blood cells from E12.5 embryos. Deformed shape observed in $\operatorname{Run} x 1^{-/}$erythrocytes (see Figure 1B) was rarely seen in $R u n x 1^{-1}:: G 1-H R D-R u n x 1$ erythrocytes. 

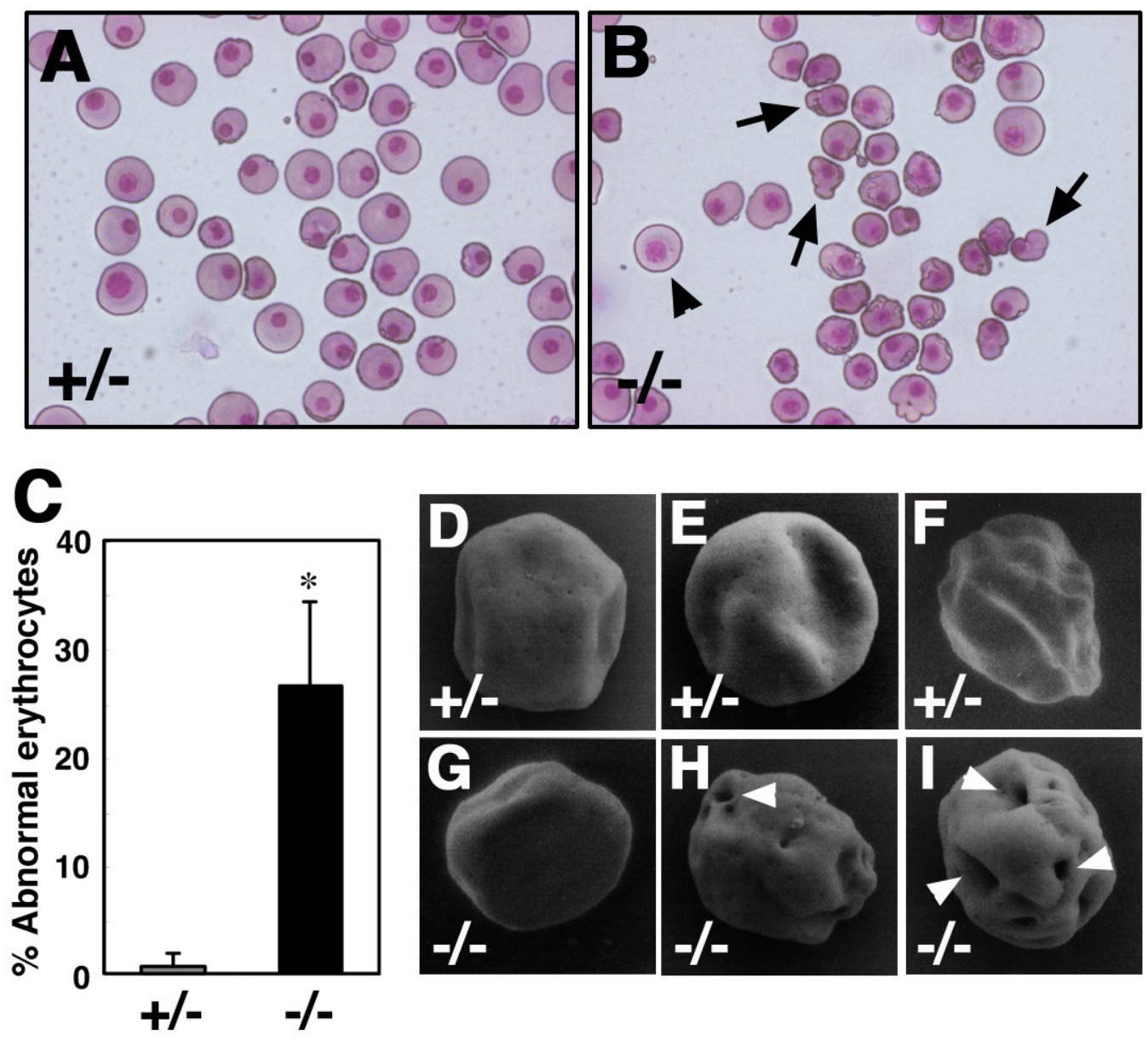
A

EryP (E8.5)

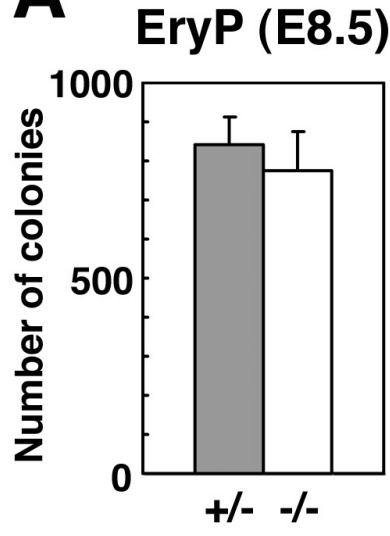

B Benzidine (E8.5)
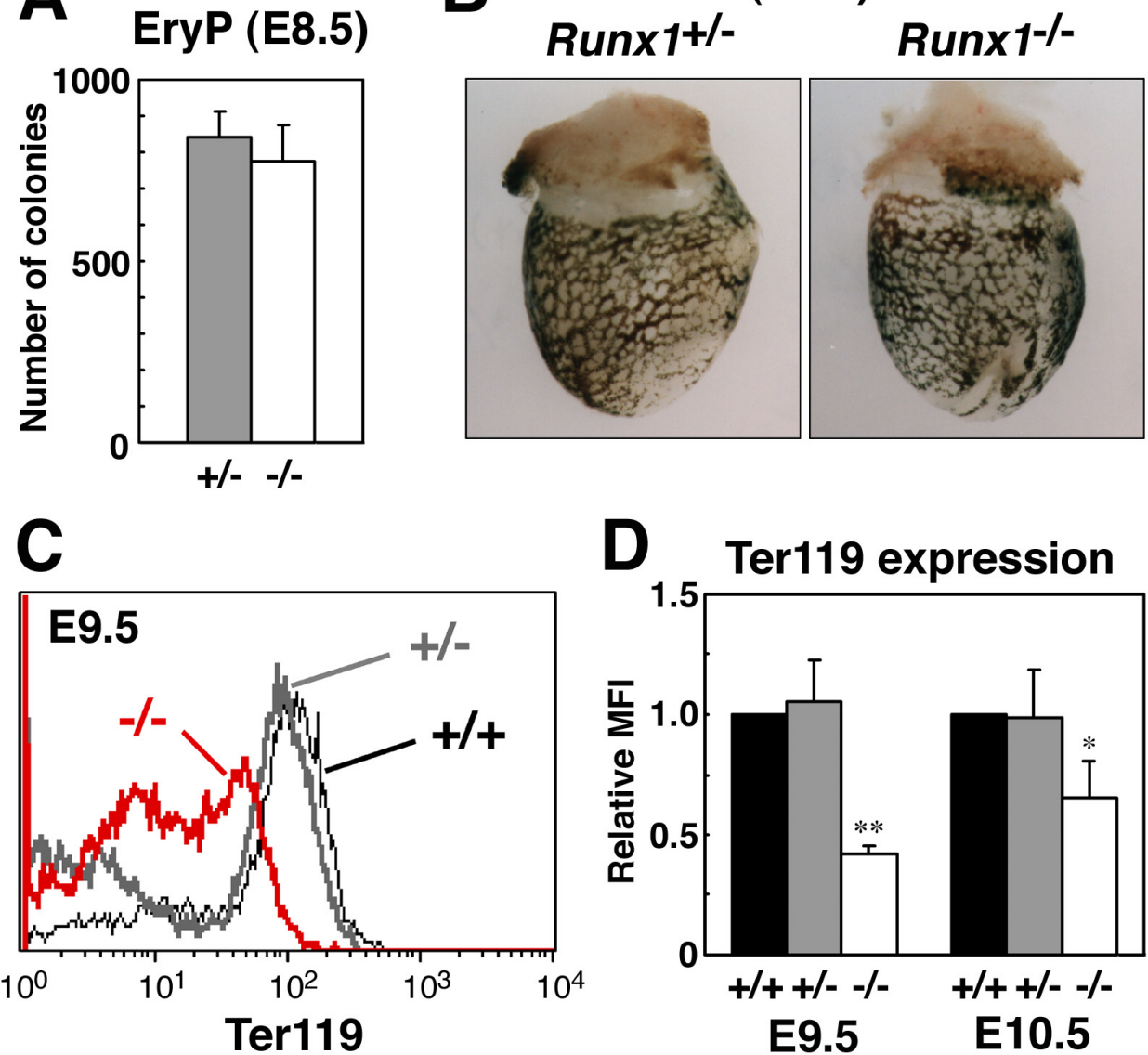

D Ter119 expression

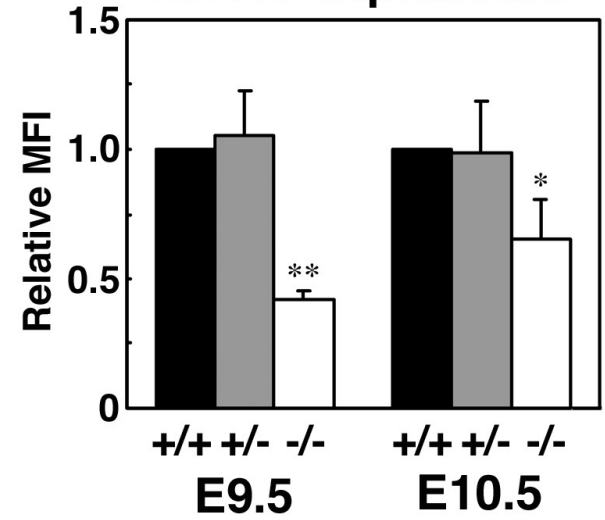




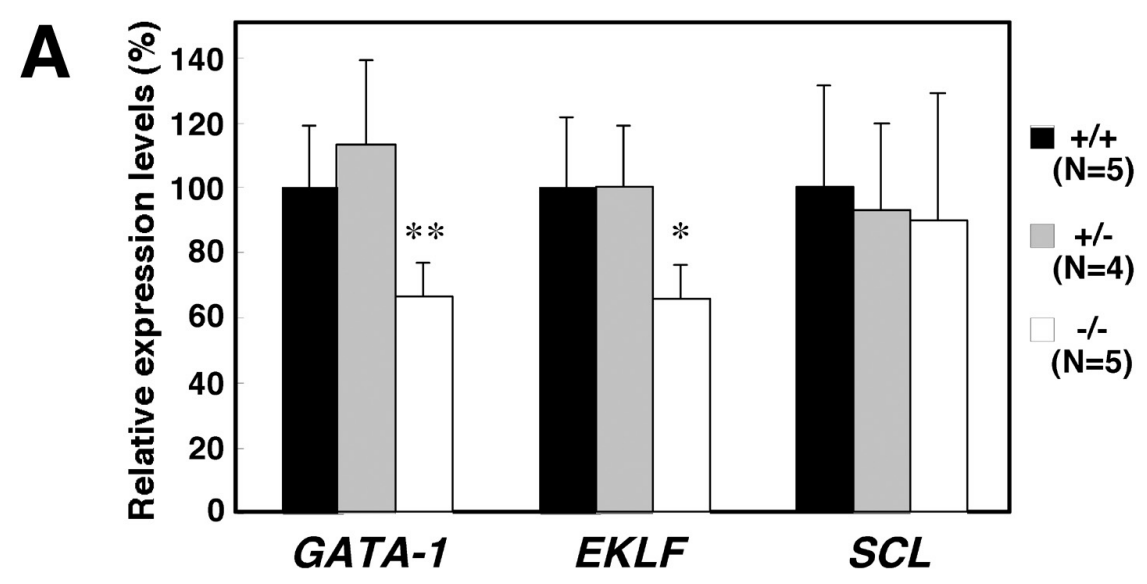

B

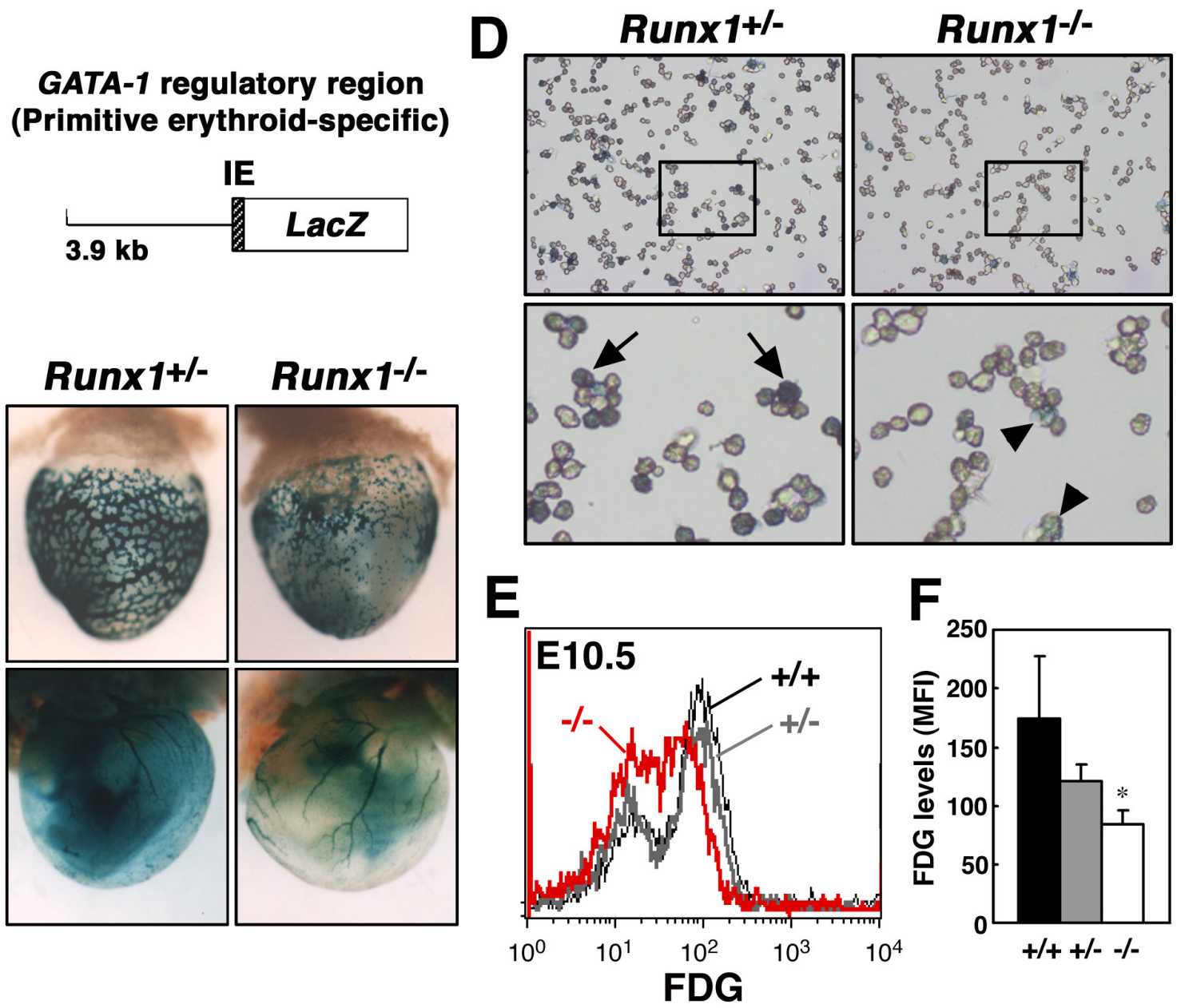


A GATA-1 regulatory region

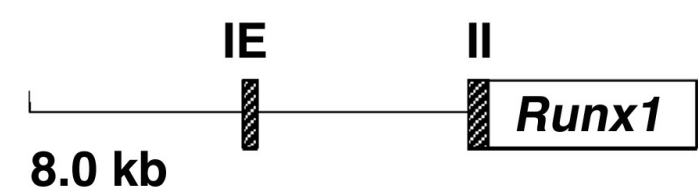

B

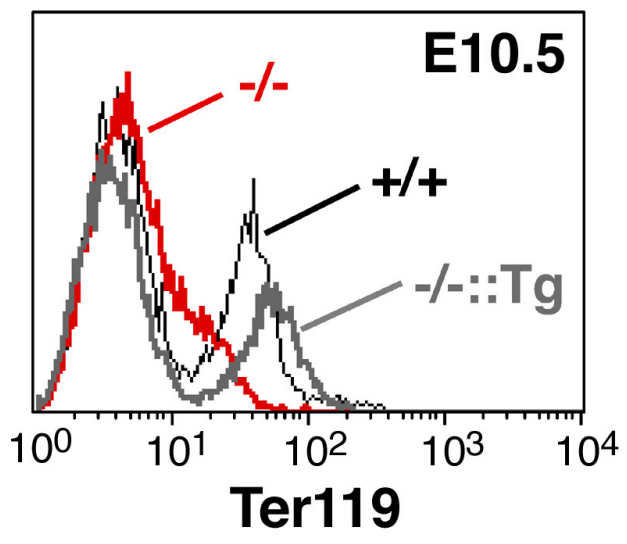

C $\begin{aligned} & \text { Ter119 } \\ & \text { expression }\end{aligned}$

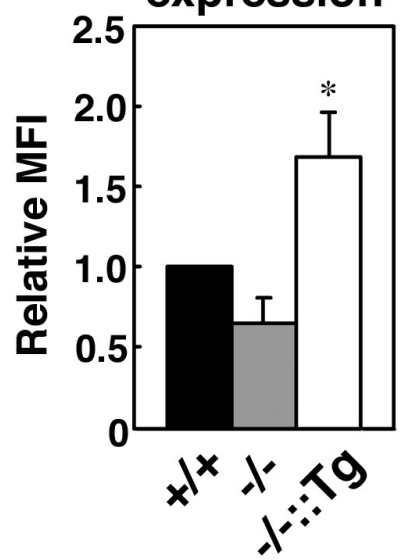

D

Runx1+/-

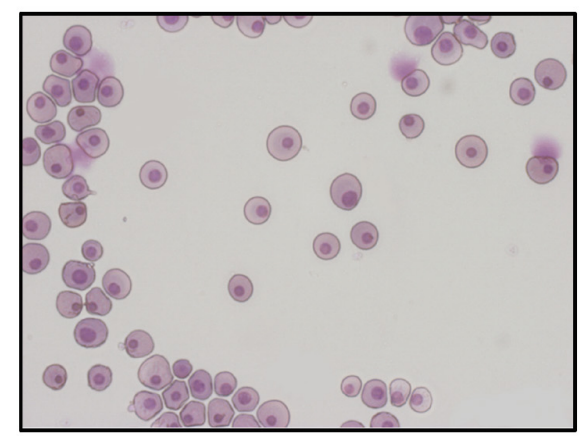

Runx1-/-::Tg

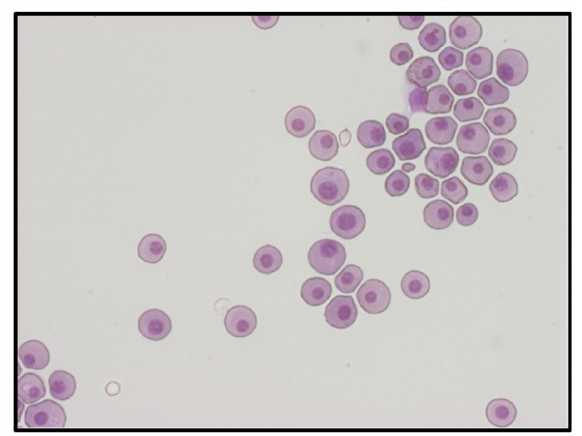

